\title{
First order phase transition between two centro-symmetric superradiant crystals
}

\author{
Xiangliang Li $\odot,{ }^{1}$ Davide Dreon $\odot,{ }^{1}$ Philip Zupancic $\odot,{ }^{1}$ Alexander Baumgärtner $\odot,{ }^{1}$ Andrea Morales $\odot,{ }^{1}$ Wei Zheng $\odot,{ }^{2,3,4}$ \\ Nigel R. Cooper $\odot,{ }^{2}$ Tobias Donner $\odot,{ }^{1, *}$ and Tilman Esslinger ${ }^{1}$ \\ ${ }^{1}$ Institute for Quantum Electronics, Eidgenössische Technische Hochschule Zürich, Otto-Stern-Weg 1, 8093 Zurich, Switzerland \\ ${ }^{2}$ T.C.M. Group, Cavendish Laboratory, University of Cambridge, J.J. Thomson Avenue, Cambridge CB3 OHE, United Kingdom \\ ${ }^{3}$ Hefei National Laboratory for Physical Sciences at the Microscale and Department of Modern Physics, \\ University of Science and Technology of China, Hefei 230026, China \\ ${ }^{4}$ CAS Center for Excellence in Quantum Information and Quantum Physics, \\ University of Science and Technology of China, Hefei 230026, China
}

(Received 16 April 2020; accepted 31 January 2021; published 5 March 2021)

\begin{abstract}
We observe a structural phase transition between two configurations of a superradiant crystal by coupling a Bose-Einstein condensate to an optical cavity and applying imbalanced transverse pump fields. The transition can be interpreted as a transition between two nonpolar, centro-symmetric structures involving a change in polarization. We find that this first order phase transition is accompanied by transient dynamics of the order parameter which we measure in real time. The phase transition and the excitation spectrum can be derived from a microscopic Hamiltonian, in quantitative agreement with our experimental data.
\end{abstract}

DOI: 10.1103/PhysRevResearch.3.L012024

Structural phase transitions between different crystal configurations play an important role in the description of materials. They arise from a delicate balance of competing internal forces and can be complex to describe owing to their intrinsically nonlinear character. The study of the transition dynamics is especially challenging due to the very short timescales determining the process in solid-state systems [1-4]. Beyond condensed-matter systems, quantum structural phase transitions have also been studied in ion crystals [5-7] at effectively zero temperature.

In quantum simulations with ultracold atoms loaded into optical lattices [8,9], the lattice structure is dictated by the externally applied laser fields, which is the strength but also limitation of this approach. For example, the crystallization process itself, or a structural phase transition between different crystal configurations, cannot be studied. Such phenomena can, however, be addressed with dynamical lattice potentials that emerge inside optical cavities coupled to driven atoms [10].

In such many-body cavity QED settings, atoms are placed into an initially unoccupied cavity mode and illuminated by an external pump laser field. Above a critical pump strength, the atoms lower the total energy of the system by crystallizing into a superradiant pattern that supports Bragg scattering of photons into the cavity mode. The interference between the classical pump laser field and the emerging

\footnotetext{
*donner@phys.ethz.ch

Published by the American Physical Society under the terms of the Creative Commons Attribution 4.0 International license. Further distribution of this work must maintain attribution to the author(s) and the published article's title, journal citation, and DOI.
}

self-consistent quantized cavity field then gives rise to a dynamic potential that enforces the atomic pattern formation. This approach has been used to study diverse aspects of crystallization phase transitions between an initially unordered phase and a superradiant crystal [10-16], as well as dynamic instabilities in ring cavity configurations $[10,17,18]$. Yet first order structural phase transitions between two superradiant configurations of distinct geometry have not been observed.

The challenge in implementing such transitions in manybody cavity QED simulations lies in the fact that multiple cavity modes need to be involved [19]. In this Letter, we demonstrate that a structural phase transition can also be induced in an experimentally simpler way by coupling to both quadratures of a single cavity mode. Monitoring the light field leaking out of the cavity, we observe the associated rapid jump, the oscillation, and the relaxation of the order parameter.

In our experiment, we induce a first order phase transition between two different emergent crystalline configurations that arise in the atomic density of a Bose-Einstein condensate (BEC) coupled to a high-finesse optical cavity. The two crystal structures correspond to distinct minima in the free energy of the system (Fig. 1). Both structures are centrosymmetric, each one breaking a discrete $\mathbb{Z}_{2}$ symmetry, and have different symmetries of the wave function. The difference between these structures can be directly connected to the concept of polarization developed in the so-called modern theory of polarization [20-22]: Interpreting the atomic density as an electronic density in a real crystal, the centro-symmetric nature of the structures implies that both are nonpolar. A transition between the two structures corresponds to a shift of the symmetry center as shown in Fig. 1. In a real crystal, such a shift always comes with a corresponding current proportional 
(a)

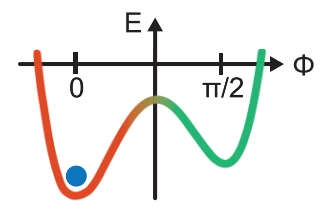

$\mathrm{SR}_{1}$

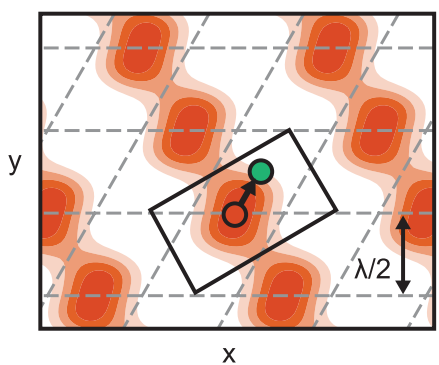

(b)

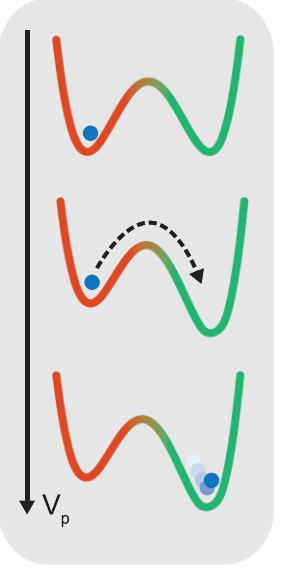

(c)
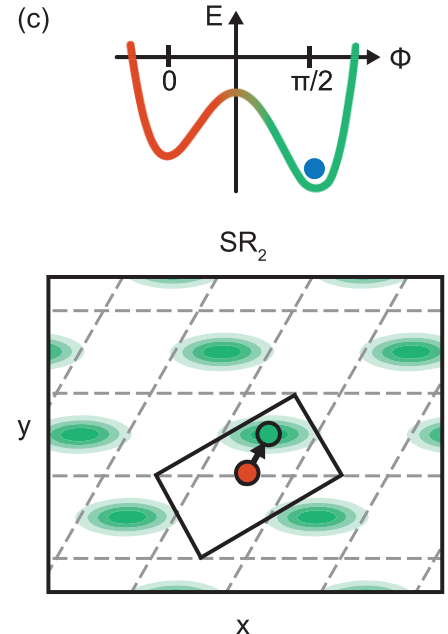

(d)

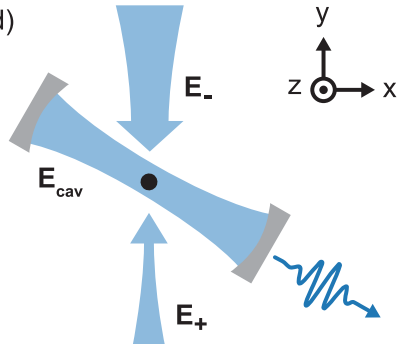

(e)

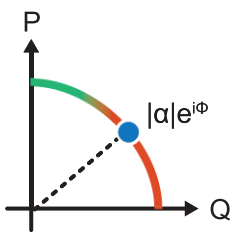

FIG. 1. (a) and (c) The free energy $E$ as a function of the phase $\phi$ of the intracavity light field supports two distinct minima. They correspond to two crystal structures, $\mathrm{SR}_{1}$ and $\mathrm{SR}_{2}$, with different symmetries as sketched in real space in red and green, where dark colors indicate high atomic densities. The black rectangle indicates the primitive cell of the lattice. The colored circles indicate the symmetry centers of the respective structures, and the arrows highlight the shift in position. Dashed gray lines indicate the maxima of the individual standing wave light fields. (b) As the free energy deforms during the experimental sequence, local and global minima swap, and the system undergoes a first order phase transition. The excess energy from the metastable state results in a damped oscillation of the order parameter around the new global minimum. (d) Two imbalanced counterpropagating pump beams $\mathbf{E}_{ \pm}$couple the BEC to quadratures $Q$ and $P$ of the intracavity electric field $\mathbf{E}_{c}$. Each quadrature generates a different interference pattern of the electric fields. (e) Using a heterodyne detector, we measure the amplitude $|\alpha|$ and phase $\phi$ of the light field leaking from the cavity. From the phase we reconstruct to which quadrature the atoms are coupled and hence which crystal structure they acquire.

to the change in polarization. In our case this current is given by the shift of the atomic patterns.

The microscopic origin of the two structures can be understood from the interaction between atoms and light, captured by the Hamiltonian $\hat{H}_{A-L}=-\alpha_{s} \hat{\mathbf{E}}^{\dagger} \cdot \hat{\mathbf{E}}$, where $\alpha_{s}$ is the scalar atomic polarizability and $\hat{\mathbf{E}}$ is the total electric field. The BEC is placed at the cavity mode center and exposed to an off-axis pump laser beam [see Fig. 1(d)]. The total electric field is the sum of the cavity field $\hat{\mathbf{E}}_{c}$ and the pump field $\mathbf{E}_{p}, \hat{\mathbf{E}}=\hat{\mathbf{E}}_{c}+\mathbf{E}_{p}$. The cavity is initially in the vacuum state but can be populated by Raman processes where photons are scattered via the atoms from the pump into the cavity and vice versa. In order to scatter light constructively, the atoms have to organize in a periodic structure that obeys the Bragg condition and comes at the cost of kinetic energy. This is thus possible only above a critical pump power, where the overall energy is lowered by atomic self-organization [10,23], and the system becomes superradiant. At the phase transition, the BEC spontaneously breaks a discrete translational symmetry [24].

In contrast to previous self-organization studies that relied on either a standing wave $[11,12,14,16]$ or a running wave [25-27] pump beam, we employ two unbalanced counterpropagating beams [28], that is, $\mathbf{E}_{p}=\left(\mathbf{E}_{+}+\mathbf{E}_{-}\right) / 2$, with $\mathbf{E}_{ \pm}=$ $E_{ \pm} e^{ \pm i \mathbf{k}_{p} \mathbf{r}} \mathbf{e}_{z}$. Here, $E_{ \pm}$are the electric field amplitudes, $\mathbf{e}_{z}$ is the polarization vector, $\mathbf{k}_{p}$ is the wave vector with $\left|\mathbf{k}_{p}\right|=2 \pi / \lambda$, and $\lambda$ is the wavelength of the light. The beam in the $-\mathbf{k}_{p}$ direction is the retroreflected $+\mathbf{k}_{p}$ beam, whose focus position allows us to tune the imbalance parameter $\gamma=\left(E_{+} / E_{-}\right)^{1 / 2}$. The two beams interfere, creating a standing wave with an offset. The standing wave corresponds to an optical lattice depth $V_{p}=-\alpha_{s} E_{+} E_{-}$, which we use as the control parameter for the phase transition.

The lattice Hamiltonian is derived from the interaction $H_{A-L}=-\alpha_{s} \hat{\mathbf{E}}^{\dagger} \cdot \hat{\mathbf{E}}$ by inserting the total electric field $\hat{\mathbf{E}}=$ $\mathbf{E}_{p}+\hat{\mathbf{E}}_{c}$ [29]. In the rotating wave approximation, the electric field of the cavity is given by $\hat{\mathbf{E}}_{c}=E_{0} \hat{a} \cos \left(\mathbf{k}_{c} \mathbf{r}\right) \mathbf{e}_{z}$, where $E_{0}$ is the electric field strength of a single cavity photon and $\hat{a}$ $\left(\hat{a}^{\dagger}\right)$ is the operator annihilating (creating) a photon in the cavity mode. The wave vector along the cavity axis is $\mathbf{k}_{c}$, with $\left|\mathbf{k}_{c}\right|=\left|\mathbf{k}_{p}\right|$. This results in

$$
\begin{aligned}
\hat{H}_{A-L}(\mathbf{r})= & V_{p} \cos ^{2}\left(\mathbf{k}_{p} \mathbf{r}\right)+\hat{V}_{c} \cos ^{2}\left(\mathbf{k}_{c} \mathbf{r}\right) \\
& +\hat{V}_{1} \cos \left(\mathbf{k}_{p} \mathbf{r}\right) \cos \left(\mathbf{k}_{c} \mathbf{r}\right)+\hat{V}_{2} \sin \left(\mathbf{k}_{p} \mathbf{r}\right) \cos \left(\mathbf{k}_{c} \mathbf{r}\right) .
\end{aligned}
$$

$\hat{V}_{c}=\hbar U_{0} \hat{a}^{\dagger} \hat{a}$ is the potential of the quantized cavity lattice, where $U_{0}$ is the single-atom dispersive shift, and

$$
\begin{aligned}
& \hat{V}_{1}=\frac{1}{2}\left(\gamma+\frac{1}{\gamma}\right)\left(\hbar U_{0} V_{p}\right)^{1 / 2}\left(\hat{a}+\hat{a}^{\dagger}\right), \\
& \hat{V}_{2}=-\frac{i}{2}\left(\gamma-\frac{1}{\gamma}\right)\left(\hbar U_{0} V_{p}\right)^{1 / 2}\left(\hat{a}-\hat{a}^{\dagger}\right)
\end{aligned}
$$

are the amplitudes of the two possible interference lattice terms between pump and cavity. The terms proportional to $\hat{V}_{1,2}$ in Eq. (1) both couple the atomic momentum state $\mathbf{k}=\mathbf{0}$ to the states $\mathbf{k}_{ \pm}=\mathbf{k}_{p} \pm \mathbf{k}_{c}$. However, they couple to spatial patterns with different parities, either $\cos \left(\mathbf{k}_{p} \mathbf{r}\right)$ or $\sin \left(\mathbf{k}_{p} \mathbf{r}\right)$. In a band structure picture, this means that $\hat{V}_{1}$ couples more strongly to the second $(p)$ band than to the first $(s)$ band and vice versa, assuming repulsive optical potentials [30]. 
Accordingly, the emerging self-organized phases will have different spatial structures, as is also shown in Figs. 1(a) and $1(b)$.

The two possible lattice configurations correspond to the two minima of the free energy in Fig. 1 and are either $\left\langle\hat{V}_{1}\right\rangle \neq 0$ and $\left\langle\hat{V}_{2}\right\rangle=0$ or $\left\langle\hat{V}_{2}\right\rangle \neq 0$ and $\left\langle\hat{V}_{1}\right\rangle=0$. They will be referred to as superradiant phases $\mathrm{SR}_{1}$ and $\mathrm{SR}_{2}$, respectively. Using the point of spatial inversion symmetry of the lattice structure of $\mathrm{SR}_{1}$ as the origin $[\mathbf{r}=(0,0)]$, the spatial inversion reference point for $\mathrm{SR}_{2}$ is shifted to $\mathbf{r}^{\prime}=(\sqrt{3} / 2-2,1 / 2) \pi /\left|\mathbf{k}_{p}\right|$.

All potentials except $V_{p}$ have a nonzero value only in the superradiant phases, i.e., when $\langle\hat{a}\rangle \neq 0$. Specifically, $\hat{V}_{1}$ and $\hat{V}_{2}$ are proportional to the orthogonal quadratures $Q=$ $\frac{1}{\sqrt{2}}\left\langle\hat{a}^{\dagger}+\hat{a}\right\rangle$ and $P=\frac{i}{\sqrt{2}}\left\langle\hat{a}^{\dagger}-\hat{a}\right\rangle$ of the cavity field, respectively [31]. Therefore, using the complex-valued expectation value of the intracavity field $\langle\hat{a}\rangle=\alpha=|\alpha| e^{i \phi}$ as an order parameter, it is possible not only to observe the transition to a superradiant phase but also to distinguish $\mathrm{SR}_{1}$ from $\mathrm{SR}_{2}$ via the phase of the light field. In the chosen reference frame rotating with the pump field, either the real or the imaginary quadrature of the cavity field will be occupied in $\mathrm{SR}_{1}$ or $\mathrm{SR}_{2}$, respectively.

We prepare a BEC of $N=4.4(4) \times 10^{5}{ }^{87} \mathrm{Rb}$ atoms and couple it dispersively to a single mode of our optical cavity [29]. The pump beams have a wavelength of $\lambda=780.1 \mathrm{~nm}$, which is blue detuned with respect to the $D_{2}$ line of ${ }^{87} \mathrm{Rb}$ by $+2 \pi \times 69.8$ (1) $\mathrm{GHz}$ such that the atoms experience a repulsive potential. At this wavelength, the single-atom dispersive shift is $U_{0}=2 \pi \times 47.5(1) \mathrm{Hz}$, and the recoil frequency with one photon is $\omega_{r}=E_{r} / \hbar=2 \pi \times 3.77 \mathrm{kHz}$. We vary the pump to cavity detuning $\Delta_{c} / 2 \pi=\left(\omega_{p}-\omega_{c}\right) / 2 \pi$ in a range of 0 to $-10 \mathrm{MHz}$, where $\omega_{p}$ and $\omega_{c}$ are the frequencies of the pump beam and bare-cavity resonance, respectively. The two counterpropagating pump beams are incident on the atoms at an angle of $60(1)^{\circ}$ with respect to the cavity mode.

We record phase diagrams as a function of detuning $\Delta_{c}$ and pump lattice depth $V_{p}$ in the following way. We initially fix the relative coupling strengths of the two counterpropagating pump beams by choosing $\gamma=1.28(3)$. We linearly ramp up the pump beam lattice depth $V_{p}$ from 0 to $36(3) E_{r}$ in $50 \mathrm{~ms}$ and repeat the same experimental sequence for different values of $\Delta_{c}$. We record the light field leaking out of the cavity via a heterodyne detection setup and extract the field amplitude $|\alpha|$ and the phase $\phi$ as functions of $V_{p}$ and $\Delta_{c}$. The resulting phase diagrams [Fig. 2(a)] show three different phases, the normal (superfluid) phase (SF) and the superradiant phases $\mathrm{SR}_{1}$ and $\mathrm{SR}_{2}$. In the SF phase, we detect only the vacuum noise of the cavity mode. In phases $\mathrm{SR}_{1}$ and $\mathrm{SR}_{2}$, nonzero average intracavity fields are detected. While the phase $\mathrm{SR}_{2}$ extends to large lattice depths $V_{p}$, phase $\mathrm{SR}_{1}$ has a finite extent for nonzero cavity detunings due to the parity of the Hamiltonian for positive atomic detuning, where the cavity-atom coupling gets counteracted by the growing band gap of the pump lattice. Self-organization by coupling to the $p$ band becomes energetically unfavorable above a certain lattice depth [30]. In the red pump detuning case, $\mathrm{SR}_{1}$ will extend to large lattice depths, and $\mathrm{SR}_{2}$ is always unfavored. Blue pump detuning giving a limited extent to $\mathrm{SR}_{1}$ is thus necessary to make $\mathrm{SR}_{2}$ possible.

The $\pi / 2$ difference in the phase of the cavity field between $\mathrm{SR}_{1}$ and $\mathrm{SR}_{2}$ [Fig. 2(b)] is a consequence of the coupling
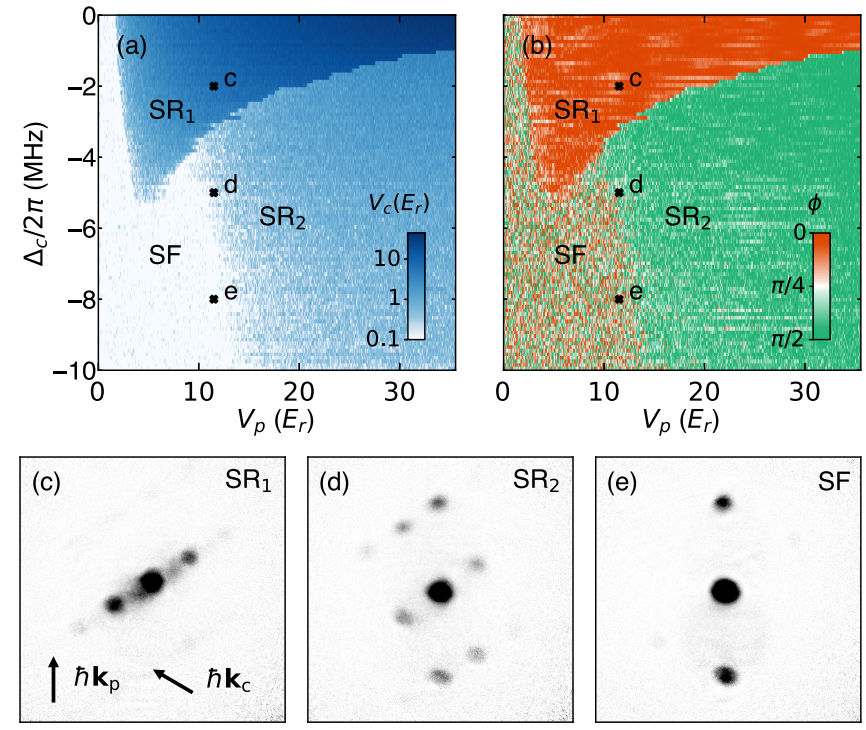

FIG. 2. (a) and (b) Phase diagram showing the complex order parameter as the expectation value $\langle\hat{a}\rangle=|\alpha| e^{i \phi}$ of intracavity field, measured as a function of the pump lattice depth $V_{p}$ and the cavity detuning $\Delta_{c}$. From the amplitude $|\alpha|$ we extract the intracavity lattice depth $\left\langle\hat{V}_{c}\right\rangle=V_{c}$, shown in (a). At the phase transition between the two superradiant phases $V_{c}$ changes abruptly. (b) Phase of the light field mapped to the first quadrant $(\phi \in[0, \pi / 2])$ to highlight the $\pi / 2$ phase jump. (c), (d), and (e) Absorption images of the atomic cloud after ballistic expansion, showing the momentum distribution of the atoms. The images are recorded at $V_{p}=12(1) E_{r}$ and $\Delta_{c} / 2 \pi=$ $-2,-5,-8 \mathrm{MHz}$, as indicated in the phase diagrams. Dark areas show high atomic densities. (c) and (d) correspond to the atomic density distribution of Figs. 1(a) and 1(c), respectively. The arrows denote the pump and cavity wave vectors $\hbar \mathbf{k}_{p}$ and $\hbar \mathbf{k}_{c}$.

to two orthogonal quadratures, corresponding to the two interference terms in Eq. (1), each representing one of the two crystal structures (see Fig. 1). As shown in Figs. 2(c), 2(d), and 2(e), the difference also appears in time-of-flight images, where one records the momentum distribution of the atoms. In the normal phase, only the two momenta at $\pm 2 \hbar \mathbf{k}_{p}$, associated with the $\lambda / 2$ periodicity of the pump lattice, are visible besides the zero-momentum mode [see Fig. 2(e)]. In $\mathrm{SR}_{1}$, these momentum components are suppressed, but the momenta $\pm \hbar\left(\mathbf{k}_{p}-\mathbf{k}_{c}\right)$ are populated, indicating a dominantly one-dimensional density modulation [see Fig. 2(c)]. In $\mathrm{SR}_{2}$, two additional nonparallel momenta $\pm \hbar\left(\mathbf{k}_{p}+\mathbf{k}_{c}\right)$ are macroscopically populated, which results in an emergent twodimensional modulation.

In the transition from $\mathrm{SR}_{1}$ to $\mathrm{SR}_{2}$, the discontinuity of the order parameter jumping between two nonzero values is the first indication of a first order phase transition [32]. We monitor and plot the amplitude and phase of the cavity field as functions of time in Figs. 3(a) and 3(b) for different values of $\Delta_{c}$. In addition to the abrupt change in both observables at the phase transition, we record an oscillation of the phase $\phi$ after the transition. It has a single frequency that depends on $\Delta_{c}$ and $V_{p}$ and decays within a few oscillation periods [see Figs. 3(b) and 3(d)].

Our observations can be understood as a transition from a metastable state to the ground state. We numerically calculate 

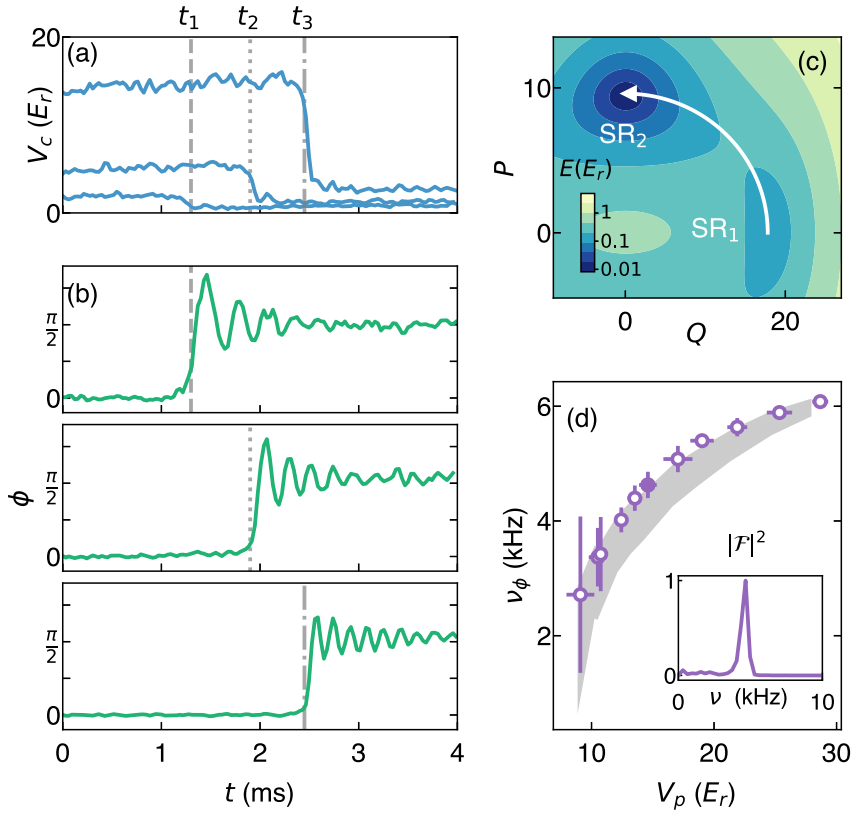

FIG. 3. Phase transition and relaxation of the order parameter. (a) and (b) Cuts of $V_{c}$ and the $\phi$ phase diagram [similar to Figs. 2(a) and 2(b) but for $\gamma=1.37(5)]$, respectively, for different cavity detunings $\Delta_{c} / 2 \pi=-3.75,-2.75,-1.75 \mathrm{MHz}$, showing a jump at the phase transition. The transition points $t_{1,2,3}$ correspond, respectively, to $V_{p}=11(1) E_{r}, 15(1) E_{r}, 25(2) E_{r}$. (c) The energy landscape [calculated from the parameters of the solid data point in (d)] truncated to the photonic space shows two minima on the different quadrature axes. The system jumps from the local minimum to the global minimum when the phase transition happens. We extract the normal mode frequencies from the curvatures of the energy landscape at the global minimum (see the Supplemental Material), which is plotted as the theoretical prediction in (d). (d) We take the Fourier transform of the time trace of the oscillating phase and extract the dominant frequency. The resulting frequencies are shown as a function of $V_{p}$ and accordingly changing $\Delta_{c}$, following the phase boundary between $\mathrm{SR}_{1}$ and $\mathrm{SR}_{2}$, and are compared to the numerical model. The gray shading shows the theoretical prediction including the experimental uncertainties of the imbalance parameter. The Fourier spectrum of the solid data point is shown in the inset. Errors indicate statistical deviation.

the energy landscape of the system from the Hamiltonian [29]. It is plotted in Fig. 3(c) as a function of the cavity field quadratures. There are two different minima located at the quadrature axes, corresponding to the two structural phases. Small changes in system parameters can turn the local minimum into a global minimum and vice versa. After ramping a control parameter, the system thus can temporarily be in the local minimum but will eventually fall into the global minimum. The oscillation in the phase of the light field after the transition reveals a collective excitation in $\mathrm{SR}_{2}$, induced by the energy difference between the minima for $\mathrm{SR}_{1}$ and $\mathrm{SR}_{2}$ when the transition takes place. We compare the oscillation frequencies with the expected frequencies of the phase mode in $\mathrm{SR}_{2}$ calculated from the curvature of the energy landscape, which shows good quantitative agreement [see Fig. 3(d)]. Different mechanisms can be envisioned driving the system from one local minimum into the other. The energetic land- (a)
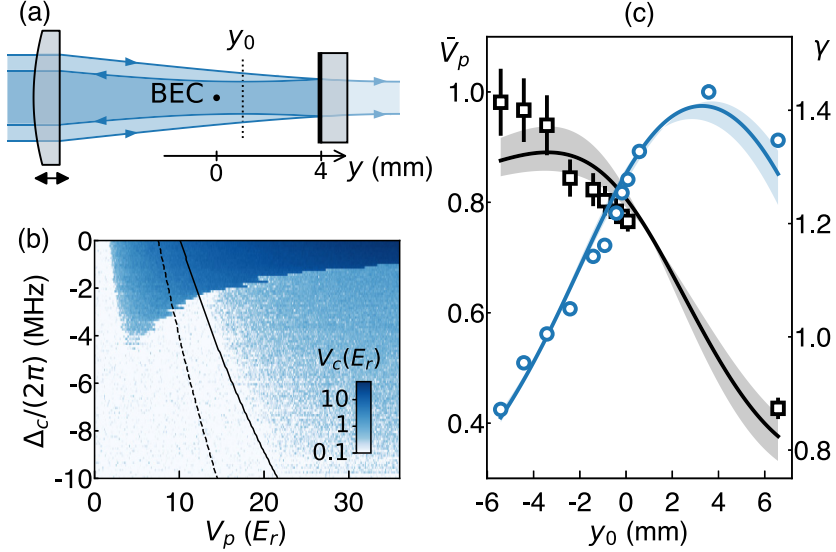

FIG. 4. Tuning the imbalance parameter $\gamma$. (a) Geometry of the system. We choose the BEC position as the origin of the $y$ axis and define $y_{0}$ as the coordinate of the beam focus. Moving the lens in the $y$ direction translates the focus and allows tuning of $\gamma$. The mirror has a finite reflectivity, which corresponds to a minor shift in $y$ of the balanced point $\gamma=1$. (b) We extract $\gamma$ from the experimental data by comparing the threshold with numerical calculations. This phase diagram is consistent with an imbalance parameter $\gamma=1.22(2)$, which gives the calculated phase boundary of $\mathrm{SR}_{2}$ shown as the solid curve. The dashed line is instead the calculated phase boundary for $\gamma=1.28(3)$ as in Fig. 2. (c) Dependence of the pump lattice depth (blue curve) and imbalance parameter $\gamma$ (black curve) on the beam focus coordinate, calculated from the geometrical model of a retroreflected Gaussian beam. The normalized pump lattice depth $\bar{V}_{p}$ is relative to the maximum measured value of $V_{p}$. Shaded areas account for the $5 \%$ systematic error of the beam waist measurement. The normalized pump lattice depths (blue circles) are measured by performing Raman-Nath diffraction calibration for different $y_{0}$ values. The imbalance parameters $\gamma$ (black squares) are extracted as in (b). Error bars account for the standard errors of the least squares fits and are smaller than the symbol size for the blue data.

scape will pass a spinodal point forcing the transition since the local minimum is disappearing. Most likely, the transition will, however, be triggered already before the spinodal point is reached since the system can overcome a finite energetic barrier either by tunneling, by thermal activation, or by additional fluctuations due to the openness of the system [33]. A comparison between the experimentally observed phase boundary and numerically calculated phase diagrams indeed suggests that such mechanisms play an important role (see Fig. S2 in the Supplemental Material [29]). Pinning down the dominant process goes, however, beyond the scope of the current publication.

The properties of the synthetic material we generated can be tuned widely, which can affect the phase diagram in both a quantitative way and a qualitative way. Our setup allows us to continuously tune the imbalance parameter $\gamma$ by moving the lens focusing the pump beam onto the atoms (see Fig. 4(a) and [29]). The expected value of $\gamma$ can be calculated using Gaussian optics [see Fig. 4(c)]. We also calculate the lattice depth $V_{p}$ as a function of the lens position and compare it with Raman-Nath diffraction measurements. The highest lattice depth occurs as expected where the laser beam has its focus on the mirror. 
So far, we have chosen $\gamma$ in a range where competition between both structures appears. However, for smaller values of the imbalance parameter, the phase $\mathrm{SR}_{2}$ shifts to larger pump lattice strengths, such that the first order phase transition effectively vanishes since $\mathrm{SR}_{1}$ and $\mathrm{SR}_{2}$ separate. We record phase diagrams for different values of $\gamma$ and observe the shift of the phase boundaries [see Fig. 4(b)]. Using the numerical results from our theoretical model [29], we fit the phase diagram and extract a measured value for $\gamma$. Calculated and fitted values of $\gamma$ are displayed in Fig. 4(c). Quantitative agreement eventually is limited by beam imperfections.

In conclusion, we explored a first order phase transition between two configurations of a self-organized crystal coupling to a single mode of an optical cavity. The real-time access to the intracavity field allowed us to study the relaxation behavior of this nonadiabatic structural phase transition. Our work demonstrates that quantum simulations with ultracold atoms not only provide conceptual insights into the electronic properties of a material $[8,9]$ but, as we demonstrated here, can also be used to study lattice distortions and structural phase transitions. The extension of our work to insulating regimes of dynamic bosonic or fermionic Hubbard models [34] in lattices exhibiting the same symmetries is possible. In that case, the atoms can be described by localized
Wannier functions instead of the delocalized wave function of a Bose-Einstein condensate. An adiabatic displacement of the atoms then leads to a polarization current that is linked to the change in Berry connection of the filled band, the integral of which plays the role of a Zak phase that can be detected in the phase of the light field leaking from the cavity [35]. Another direction for future experiments is to use multiple pump beam modes in order to realize complex crystalline structures, also featuring the formation of domains. This approach would be complementary to the use of multimode resonators [19].

Note added. Recently, Ref. [36], which theoretically discusses a related scheme coupling two density waves of a Bose-Einstein condensate to two quadratures of a cavity field, was published.

We gratefully acknowledge the support provided by A. Frank with the electronics and a valuable discussion with $\mathrm{N}$. Spaldin. We acknowledge funding from SNF, Projects No. 182650 and No. 175329 (NAQUAS QuantERA) and NCCR QSIT; from EU Horizon 2020, ERCadvanced grant TransQ (Project No. 742579) and ITN grant ColOpt (Project No. 721465); from SBFI (QUIC, Contract No. 15.0019); from EPSRC Grants No. EP/P009565/1 and No. EP/K030094/1; and from an Investigator Award of the Simons Foundation.

X.L. and D.D. contributed equally to this work.
[1] P. Beaud et al., A time-dependent order parameter for ultrafast photoinduced phase transitions, Nat. Mater. 13, 923 (2014).

[2] S. L. Johnson et al., Femtosecond Dynamics of the Collinear-toSpiral Antiferromagnetic Phase Transition in CuO, Phys. Rev. Lett. 108, 037203 (2012).

[3] M. Eichberger, H. Schäfer, M. Krumova, M. Beyer, J. Demsar, H. Berger, G. Moriena, G. Sciaini, and R. J. D. Miller, Snapshots of cooperative atomic motions in the optical suppression of charge density waves, Nature (London) 468, 799 (2010).

[4] R. Yusupov, T. Mertelj, V. V. Kabanov, S. Brazovskii, P. Kusar, J.-H. Chu, I. R. Fisher, and D. Mihailovic, Coherent dynamics of macroscopic electronic order through a symmetry breaking transition, Nat. Phys. 6, 681 (2010).

[5] I. Waki, S. Kassner, G. Birkl, and H. Walther, Observation of Ordered Structures of Laser-Cooled Ions in a Quadrupole Storage Ring, Phys. Rev. Lett. 68, 2007 (1992).

[6] M. G. Raizen, J. M. Gilligan, J. C. Bergquist, W. M. Itano, and D. J. Wineland, Ionic crystals in a linear Paul trap, Phys. Rev. A 45, 6493 (1992).

[7] E. Shimshoni, G. Morigi, and S. Fishman, Quantum structural phase transition in chains of interacting atoms, Phys. Rev. A 83, 032308 (2011).

[8] M. Lewenstein, A. Sanpera, and V. Ahufinger, Ultracold Atoms in Optical Lattices (Oxford University Press, Oxford, 2012), p. 479 .

[9] I. Bloch, J. Dalibard, and W. Zwerger, Many-body physics with ultracold gases, Rev. Mod. Phys. 80, 885 (2008).

[10] H. Ritsch, P. Domokos, F. Brennecke, and T. Esslinger, Cold atoms in cavity-generated dynamical optical potentials, Rev. Mod. Phys. 85, 553 (2013).
[11] A. T. Black, H. W. Chan, and V. Vuletić, Observation of Collective Friction Forces due to Spatial Self-Organization of Atoms: From Rayleigh to Bragg Scattering, Phys. Rev. Lett. 91, 203001 (2003).

[12] K. Baumann, C. Guerlin, F. Brennecke, and T. Esslinger, Dicke quantum phase transition with a superfluid gas in an optical cavity, Nature (London) 464, 1301 (2010).

[13] D. Schmidt, H. Tomczyk, S. Slama, and C. Zimmermann, Dynamical Instability of a Bose-Einstein Condensate in an Optical Ring Resonator, Phys. Rev. Lett. 112, 115302 (2014).

[14] J. Klinder, H. Keßler, M. Wolke, L. Mathey, and A. Hemmerich, Dynamical phase transition in the open Dicke model, Proc. Natl. Acad. Sci. USA 112, 3290 (2015).

[15] J. Léonard, A. Morales, P. Zupancic, T. Esslinger, and T. Donner, Supersolid formation in a quantum gas breaking a continuous translational symmetry, Nature (London) 543, 87 (2017).

[16] A. J. Kollár, A. T. Papageorge, V. D. Vaidya, Y. Guo, J. Keeling, and B. L. Lev, Supermode-density-wave-polariton condensation with a Bose-Einstein condensate in a multimode cavity, Nat. Commun. 8, 14386 (2017).

[17] N. Piovella, M. Gatelli, and R. Bonifacio, Quantum effects in the collective light scattering by coherent atomic recoil in a Bose-Einstein condensate, Opt. Commun. 194, 167 (2001).

[18] S. Slama, S. Bux, G. Krenz, C. Zimmermann, and P. W. Courteille, Superradiant Rayleigh Scattering and Collective Atomic Recoil Lasing in a Ring Cavity, Phys. Rev. Lett. 98, 053603 (2007).

[19] S. Gopalakrishnan, B. L. Lev, and P. M. Goldbart, Emergent crystallinity and frustration with Bose-Einstein condensates in multimode cavities, Nat. Phys. 5, 845 (2009). 
[20] R. Resta, Macroscopic polarization in crystalline dielectrics: The geometric phase approach, Rev. Mod. Phys. 66, 899 (1994).

[21] R. Resta and D. Vanderbilt, Theory of Polarization: A Modern Approach, in Physics of Ferroelectrics, Topics in Applied Physics (Springer, Berlin, Heidelberg, 2007), Vol. 105, pp. 31-68.

[22] N. A. Spaldin, A beginner's guide to the modern theory of polarization, J. Solid State Chem. 195, 2 (2012).

[23] P. Domokos and H. Ritsch, Collective Cooling and SelfOrganization of Atoms in a Cavity, Phys. Rev. Lett. 89, 253003 (2002).

[24] K. Baumann, R. Mottl, F. Brennecke, and T. Esslinger, Exploring Symmetry Breaking at the Dicke Quantum Phase Transition, Phys. Rev. Lett. 107, 140402 (2011).

[25] K. J. Arnold, M. P. Baden, and M. D. Barrett, Self-Organization Threshold Scaling for Thermal Atoms Coupled to a Cavity, Phys. Rev. Lett. 109, 153002 (2012).

[26] S. Bux, C. Gnahm, R. A. W. Maier, C. Zimmermann, and P. W. Courteille, Cavity-Controlled Collective Scattering at the Recoil Limit, Phys. Rev. Lett. 106, 203601 (2011).

[27] H. Keßler, J. Klinder, M. Wolke, and A. Hemmerich, Steering Matter Wave Superradiance with an Ultranarrow-Band Optical Cavity, Phys. Rev. Lett. 113, 070404 (2014).

[28] F. Mivehvar, H. Ritsch, and F. Piazza, Cavity-QuantumElectrodynamical Toolbox for Quantum Magnetism, Phys. Rev. Lett. 122, 113603 (2019).

[29] See Supplemental Material at http://link.aps.org/supplemental/ 10.1103/PhysRevResearch.3.L012024 for experimental details and derivations Refs. [37-41].

[30] P. Zupancic, D. Dreon, X. Li, A. Baumgärtner, A. Morales, W. Zheng, N. R. Cooper, T. Esslinger, and T. Donner, $P$-Band Induced Self-Organization and Dynamics with Repulsively
Driven Ultracold Atoms in an Optical Cavity, Phys. Rev. Lett. 123, 233601 (2019).

[31] C. Gerry and P. Knight, Introductory Quantum Optics (Cambridge University Press, Cambridge, 2004).

[32] K. Huang, Introduction to Statistical Physics (Wiley, New York, 1987).

[33] F. Piazza, P. Strack, and W. Zwerger, Bose-Einstein condensation versus Dicke-Hepp-Lieb transition in an optical cavity, Ann. Phys. (NY) 339, 135 (2013).

[34] R. Landig, L. Hruby, N. Dogra, M. Landini, R. Mottl, T. Donner, and T. Esslinger, Quantum phases from competing short- and long-range interactions in an optical lattice, Nature (London) 532, 476 (2016).

[35] F. Mivehvar, H. Ritsch, and F. Piazza, Superradiant Topological Peierls Insulator inside an Optical Cavity, Phys. Rev. Lett. 118, 073602 (2017).

[36] J. Fan, G. Chen, and S. Jia, Atomic self-organization emerging from tunable quadrature coupling, Phys. Rev. A 101, 063627 (2020).

[37] A. Morales, D. Dreon, X. Li, A. Baumgärtner, P. Zupancic, T. Donner, and T. Esslinger, Two-mode Dicke model from nondegenerate polarization modes, Phys. Rev. A 100, 013816 (2019).

[38] H. R. Carleton and W. T. Maloney, A balanced optical heterodyne detector, Appl. Opt. 7, 1241 (1968).

[39] H. P. Yuen and V. W. S. Chan, Noise in homodyne and heterodyne detection, Opt. Lett. 8, 177 (1983).

[40] R. Stierlin, R. Bättig, P.-D. Henchoz, and H. P. Weber, Excessnoise suppression in a fibre-optic balanced heterodyne detection system, Opt. Quantum Electron. 18, 445 (1986).

[41] J. F. Holmes and B. J. Rask, Optimum optical local-oscillator power levels for coherent detection with photodiodes, Appl. Opt. 34, 927 (1995). 